\title{
대청도(옹진군)의 관속식물상
}

\author{
양종철, 박수현, 하상교 ${ }^{1}$, 이유미*
}

국립수목원 산림생물조사과, ${ }^{1}$ 운서초등학교

\section{The Flora of Vascular Plants in Daecheong Island, South Korea}

\author{
Jong Cheol Yang, Su Hyun Park, Sang Gyo $\mathrm{Ha}^{1}$ and You Mi Lee* \\ Division of Forest Biodiversity and Herbarium, Korea National Arboretum, Pocheon 487-821, Korea \\ ${ }^{1}$ Incheon Unseo Elementary School, Incheonsi 400-340, Korea
}

\begin{abstract}
Distributional surveys for vascular plants were carried out in Daecheong island, Ongjin-gun, South Korea, from 2008 to 2010. The vascular plants recognized from the island were in tatal 402 taxa that were of 90 families, 269 genus, 350 species, 3 subspecies, 43 varieties, 6 forms. Among the investigated 402 taxa, 2 Korean endemic plants, 8 rare and endangered plants which was designated by Korea Forest Service, 35 specially plants designated by the Ministry of Environment were included. The naturalized plants were identified as 40 taxa and the percentage of naturalized plants index was $9.9 \%$. In addition, an unrecorded species, Thyrocarpus glochidiatus Maxim (Boraginaceae), was recognized from the island.
\end{abstract}

Key words - Daecheong island, Endemic plants, Rare plants, Naturalized plants

\section{서 언}

대청도는 지리적으로 북위 $37^{\circ} 47^{\prime} 43^{\prime \prime} \sim 37^{\circ} 50^{\prime} 53^{\prime \prime}$ 와 동 경 $124^{\circ} 41^{\prime} 20^{\prime \prime} \sim 124^{\circ} 45^{\prime} 30^{\prime \prime}$ 에 위치하며, 행정구역상으로는 인천광역시 옹진군 대청면에 해당되고, 조사지역의 북쪽으 로는 백령도, 동쪽으로는 황해도 옹진군과 마주하고 남쪽 에는 소청도가 위치한다. 식물상 구성인자를 바탕으로 Lee and $\operatorname{Yim}(2002)$ 이 제안한 한반도의 식물구계학적 위치로 는 남해안아구의 최북단에 해당된다. 본 조사지역의 기후 를 살펴보면 2005-2007년 3 년간 연평균 기온이 $11.2^{\circ} \mathrm{C}$ 로, 최고기온 $31.3^{\circ} \mathrm{C}$, 최저기온 $-11.4^{\circ} \mathrm{C}$ 의 평균값이 나타났고 연평균 강수량은 $860 \mathrm{~mm}$, 풍속은 $5.2 \mathrm{~m} / \mathrm{s}$ 로 측정되었다. 비슷한 위도상에 위치하는 지역 중 인천과 동두천의 기후 를 비교한 결과 해안지역에 가까울수록 내륙지역에 비해 겨울 최저기온이 $5^{\circ} \mathrm{C}$ 이상 높고, 연강수량이 $500 \mathrm{~mm}$ 가량 적으며 풍속이 $3.5 \mathrm{~m} / \mathrm{s}$ 정도가 센 것으로 나타났다(Korea Meteorological Administration, 2009). 대청도는 삼각 산 $(343 \mathrm{~m})$ 을 중심으로 산지가 발달되어 있고, 한반도 서해 연안에 산재해 있는 도서 중 우리나라의 최북단으로서 북
한과 접경지역에 위치하여 군사상 매우 중요한 도서이다. 이곳에 현존하는 자연식생은 오랜 기간에 걸쳐 대부분이 경작지 조성, 신탄재, 방목 및 군작전 등의 인간간섭에 의해 유지 되어 왔다. 삼각산을 중심으로 소나무(Pinus densiflora Siebold \& Zucc.) 군락이 넓게 분포하고 있지만 6부 능선 이상에는 대부분 소사나무(Carpinus turczaninovii Hance) 군락이 우점하고 있으며, 동백나무(Camellia japonica L.) 자생지는 계층구조가 단순하고, 해안사구식생은 다른 도서 에 비해 관광객의 출입이 적어 비교적 잘 보존되어 있다. 그러나 염소 등의 방목으로 인해 일부 지역의 식물은 다소 훼손된 상태이다(Choi and Lee, 2008). 본 조사지역의 식 물상 연구는 일부 Lee and Lee(1982)와 Choi and Lee(2008) 등에 의해 이루어졌으나 증거표본 또는 전반적인 조사의 부재로 인한 자료의 정확성이 미흡하여 새로이 정리하고자 하였으며, 옹진군 대청도일대의 주요 산림식물에 대한 분 포조사를 통해 특정종별 보전 및 자원화를 위한 기초자료 를 확보하고 식물구계학적 위치에서 중요성을 파악하고자 연구를 수행하였다.

*교신저자(E-mail) : ymlee99@forest.go.kr 


\section{재료 및 방법}

대청도지역의 식물상을 파악하기 위하여 2008년 5월과 8월, 2010년 4월의 추가조사를 포함하여 3차례에 걸쳐 현 지조사를 하였다(Fig. 1). 결과의 정확성을 기하기 위해 원 칙적으로 꽃, 열매, 포자 등 생식기관이 있는 개체만을 수집 대상으로 하였다. 수집된 식물들은 건조표본(dry specimen) 또는 액침표본으로 제작하였으며 국립수목원 산림생물표 본관 식물표본실 $(\mathrm{KH})$ 에 보관하였다. 분류군의 동정은 Lee (1980, 2003), Lee(1996a, 1996b), Park(1995, 2001, 2009) 등의 도감을 이용하여 수행하였으며, 주요 특기할만한 식물 들은 Ministry of Environment(2006), Oh et al.(2005), Korea National Arboretum(2008)을 참고하였다.

\section{결과 및 고찰}

\section{관속식물상}

본 연구에서 밝혀진 소산 관속식물은 90과 269속 350종 3 아종 43 변종 6 품종으로 총 402 분류군이 확인되었다. 분 류군별로는 양치식물이 8 과 12속 13종 2 변종 15 분류군 (3.7\%), 나자식물이 1 과 2속 3종 3 분류군(0.7\%), 피자식물 의 쌍자엽식물이 69과 192속 244종 3아종 29변종 3품종 279 분류군(69.5\%), 단자엽식물이 12 과 63 속 76 종 12 변종 3 품종 105 분류군(26.1\%)으로 확인되었다(Table 1). Lee and Lee (1982)가 조사한 바에 의하면, 87과 198속 215종 29 변종 3 품종으로 총 247 분류군을 보고하였다. 이는 계절 상 7 월 중 4 일간 조사한 내용으로, 본 연구에서 조사된 분 류군들의 수와 차이가 있을 수 있고, 또한 증거표본의 언급

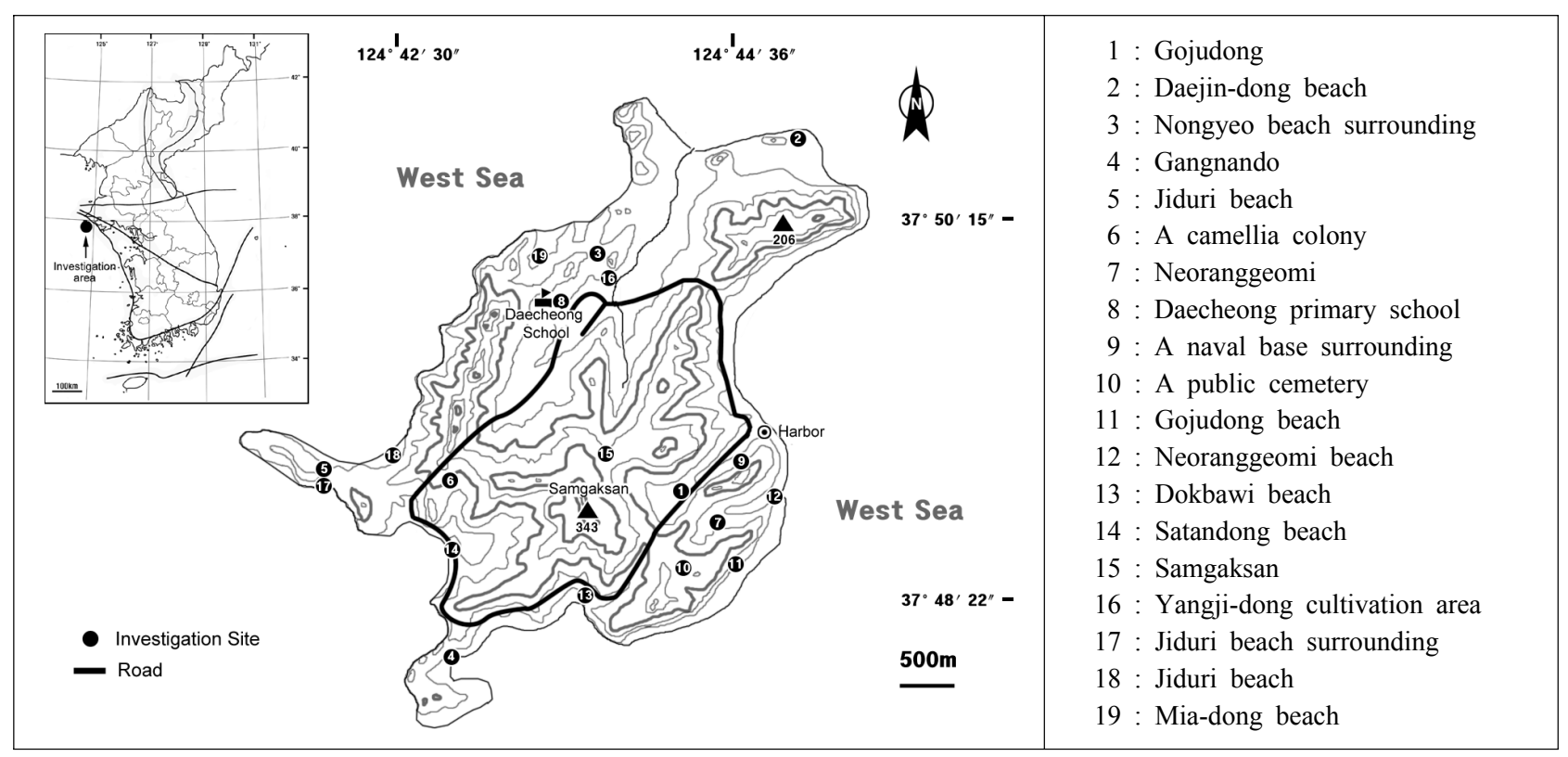

Fig. 1. Investigation routes of Daecheongdo (Ongjin-gun).

Table 1. Summary on the floristics of the Daecheongdo

\begin{tabular}{cccccccc}
\hline \hline Taxa & Fam. & Gen. & Sp. & Subsp. & Var. & For. & Total \\
\hline Pteridophyta & 8 & 12 & 13 & - & 2 & - & 15 \\
Gymnospermae & 1 & 2 & 3 & - & - & - & 3 \\
Angiospermae & & & & & & & \\
Dicotyledons & 69 & 192 & 244 & 3 & 29 & 3 & 279 \\
Monocotyledons & 12 & 63 & 76 & - & 12 & 3 & 105 \\
\hline Total & 90 & 269 & 350 & 3 & 43 & 6 & 402 \\
\hline
\end{tabular}


이 없는 이유로, 기록위주의 조사로 판단되어 비교연구에 다소 무리가 있을 것으로 생각된다. 하지만 보고 된 목록들 중 주요식물들로 솔잎란(Psilotum nudum (L.) P.Beauv.; $\mathrm{EN}, \mathrm{V}$ ), 콩짜개란(Bulbophyllum drymoglossum Maxim. ex Okubo; CR, V), 이팝나무(Chionanthus retusus Lindl. $\&$ Paxton; LC, I )가 포함되어 있어 추후 분포여부 확인 이 이루어져야 할 것으로 생각된다.

특이사항으로 동백나무군락지내에서 미기록종인 지치과의 대청지치(Thyrocarpus glochidiatus Maxim, Boraginaceae) 가 발견되었다. 본 종은 중국의 안휘성지역을 비롯하여 하 남성, 광동성, 절강성 등 동남부 지역에 주로 분포하고 있 으며, 다른 근연분류군들과 비교하여 견과에 두 층의 부속 체가 있으며, 외층은 빗살모양으로 갈라지는 특징을 갖는 다(Yang et al., 2010).

\section{한국특산식물}

조사지역의 특산식물은 4 분류군으로 서울제비꽃(Viola seoulensis Nakai), 병꽃나무(Weigela subsessilis (Nakai) L.H.Bailey) 2 분류군을 제외한 나머지 2 분류군인 개나리
(Forsythia koreana (Rehder) Nakai), 벌개미취(Aster koraiensis Nakai)는 식재된 식물로 확인되었다(Table 2).

\section{산림청지정 희귀식물}

산림청지정 희귀식물은 자료부족종(DD)을 제외한 8분 류군이 확인되었으며, 멸종위기종(CR)은 정향풀(Amsonia elliptica (Thunb.) Roem. \& Schult.), 대청부채(Iris dichotoma Pall.)가 확인 되었다. 이 두 분류군은 제한적 분포역을 가지는 종으로 계속적인 보호 및 관리가 필요할 것으로 보인다. 특히 대청부채는 대청도 서쪽 해안가의 초 지에서 주로 분포하는데 사람들의 무분별한 남획으로 인하 여 그 수가 급속도로 감소하고 있어 시급한 보호대책이 필 요하다. 그 밖에 취약종(VU)인 멱쇠채(Scorzonera austriaca subsp. glabra (Rupr.) Lipsch. \& Krasch. ex Lipsch.), 금방망이(Senecio nemorensis L.), 범부채(Belamcanda chinensis (L.) DC.) 3 분류군이 확인 되었고, 관심필요종 (LC)은 낙지다리(Penthorum chinense Pursh) 등 3 분류 군이 확인 되었다. 이는 전체 조사된 식물 중 $2.2 \%$ 를 차지 하였다(Table 3).

Table 2. The list of the Korean endemic plants

\begin{tabular}{lll}
\hline \hline Family name & \multicolumn{1}{c}{ Scientific name } & \multicolumn{1}{c}{ Korean name } \\
\hline Violaceae & Viola seoulensis Nakai & 서울제비꽃 \\
Oleaceae & Forsythia koreana (Rehder) Nakai & 개나리(식재) \\
Caprifoliaceae & Weigela subsessilis (Nakai) L.H.Bailey & 병꽃나무 \\
Compositae & Aster koraiensis Nakai & 벌개미취(식재) \\
\hline
\end{tabular}

Table 3. List of the rare and endangered plants which was designated by Korea Forest Service

\begin{tabular}{lllc}
\hline \hline Family name & \multicolumn{1}{c}{ Scientific name } & Korean name & Degree \\
\hline Apocynaceae & Amsonia elliptica (Thunb.) Roem. \& Schult. & 정향풀 & CR \\
Iridaceae & Iris dichotoma Pall. & 대청부채 & CR \\
& Scorzonera austriaca subsp. glabra (Rupr.) Lipsch. \& Krasch. & 멱쇠채 & VU \\
Compositae & ex Lipsch. & 금방망이 & VU \\
Compositae & Senecio nemorensis L. & 범부채 & VU \\
Iridaceae & Belamcanda chinensis (L.) DC. & 낙지다리 & LC \\
Crassulaceae & Penthorum chinense Pursh & 개지치 & LC \\
Boraginaceae & Lithospermum arvense L. & 두루미천남성 & LC \\
Araceae & Arisaema heterophyllum Blume & 실부추 & DD \\
Liliaceae & Allium anisopodium Ledeb. & & \\
\hline \multicolumn{2}{c}{9 taxa }
\end{tabular}

" $\mathrm{CR}$ (멸종위기종: critically endangered), $\mathrm{VU}$ (취약: vulnerable), $\mathrm{LC}$ (관심필요: least concern), $\mathrm{DD}$ (자료부족: data deficient). 


\section{식물구계학적 특정식물}

환경부지정 특정식물은 I 등급에서 $\mathrm{V}$ 등급까지 36 분류 군으로 확인되었다. 그 중 $\mathrm{V}$ 등급에 속하는 식물은 낙지다 리, 정향풀, 금방망이, 대청부채 4 분류군이 확인되었으며, III등급에는 멱쇠채, 방울비짜루(Asparagus oligoclonos Maxim.), 갯그령(Elymus mollis Trin.) 3 분류군이 조사 되었다. 결과적으로 전체 조사된 식물 중 $8.9 \%$ 를 차지하였 다(Table 4). V 등급 분류군들은 다소 제한적 분포 특성을 보이므로, 추후 자생지 보존과 관리가 필요할 것으로 생각 된다.

\section{식물지리적 분포특성}

본 조사지역에서 확인된 식물들 중 주로 중부이남에 분 포하는 남방계식물들로는 거지덩굴(Cayratia japonica (Thunb.) Gagnep.), 동백나무(Camellia japonica L.), 보춘화(Cymbidium goeringii (Rchb.f.) Rchb.f.), 후박 나무(Machilus thunbergii Siebold \& Zucc.) 등 약 7분류 군이 관찰되었으며, 대청부채(Iris dichotoma Pall.), 멱쇠 채(Scorzonera austriaca subsp. glabra (Rupr.) Lipsch. $\&$ Krasch. ex Lipsch.) 등 경기 이북에 주로 분포하는 식
물들도 함께 관찰되었다(Table 5). 본 조사지역은 식물구 계학적 위치로는 남해안아구에 속해 있으나(Lee and Yim, 2002) 관서, 중부, 남부아구의 경계가 가장 근접해 있는 지 역으로 남부지역 식물들에 있어서는 북방한계선이 되며, 북부지역의 식물들에 있어서는 남방한계선이 되는 지역이 다. 특히 대청도 후박나무의 경우 한반도내 북방한계선에 위치하게 된다. 따라서 본 조사지역은 다양한 식물들의 생 육한계를 파악하고 연구하는데 있어 중요한 지역으로 보다 세밀한 식물지리학적 연구가 필요 할 것으로 판단된다.

\section{귀화식물}

본 조사에서 확인된 귀화식물은 40 분류군으로 전체조사 식물의 $9.9 \%$ 를 차지하였다. 국화과와 벼과가 가장 많은 비 중을 차지하였으며, 십자화과, 마디풀과 등의 순으로 나타 났다(Table 6). 산지보다 대부분 논, 밭 잡초로 분포하고 있었으며, 경지이용이 높아질수록 증가율 또한 높아질 것 으로 보인다. 이 중 열대아메리카원산으로 우리나라에서는 인천 옹진군의 대청도와 백령도의 바닷가 모래땅에서 자라 고 있는 대청가시풀(Cenchrus longispinus (Hack.) Fern.) 이 가장 문제시되는 귀화식물로(Park, 2009), 총포의 열

Table 4 . The list of specially designated plants by the Ministry of Environment (only III $\sim \mathrm{V}$ degree)

\begin{tabular}{clc}
\hline \hline Degree & \multicolumn{1}{c}{ Scientific name / Korean name } & Total \\
\hline \multirow{2}{*}{ V } & $\begin{array}{l}\text { Penthorum chinense Pursh 낙지다리, Amsonia elliptica } \text { (Thunb.) Roem. \& Schult. 정향풀, } \\
\text { Senecio nemorensis L. 금방망이, Iris dichotoma Pall. 대청부채 }\end{array}$ & 4 \\
\hline \multirow{2}{*}{ III } & $\begin{array}{l}\text { Scorzonera austriaca subsp. glabra (Rupr.) Lipsch. \& Krasch. ex Lipsch. 멱쇠채, } \\
\text { Asparagus oligoclonos } \text { Maxim. 방울비짜루, Elymus mollis } \text { Trin. 갯그령 }\end{array}$ & 3 \\
\hline
\end{tabular}

Table 5. The southern and northern types of plants of the Daecheongdo

\begin{tabular}{llll}
\hline \hline Family name & \multicolumn{1}{c}{ Scientific name } & Korean name & Distribution \\
\hline Vitaceae & Cayratia japonica (Thunb.) Gagnep. & 거지덩굴 & \\
Theaceae & Camellia japonica L. & 동백나무 & \\
Orchidaceae & Cymbidium goeringii (Rchb.f.) Rchb.f. & 보춘화 & \\
Leguminosae & Caesalpinia decapetala (Roth) Alston & 실거리나무 & southern types \\
Rosaceae & Rubus hirsutus Thunb. & 장딸기 & \\
Araceae & Arisaema ringens (Thunb.) Schott & 큰천남성 & \\
Lauraceae & Machilus thunbergii Siebold \& Zucc. & 후박나무 & \\
\hline \multirow{2}{*}{ Compositae } & Scorzonera austriaca subsp. glabra (Rupr.) Lipsch. \& Krasch. & 멱쇠채 & \\
Iridaceae & ex Lipsch. & northern types \\
\hline
\end{tabular}


Table 6. The list of naturalized plants

\begin{tabular}{|c|c|c|}
\hline Family name & Scientific name / Korean name & Total \\
\hline Polygonaceae & Fallopia convolvulus (L.) A.Löve 나도닭의덩굴, Rumex crispus L. 소리쟁이 & 2 \\
\hline Caryophyllaceae & Cerastium glomeratum Thuill. 유럽점나도나물, Silene armeria L. 끈끈이대나물 & 2 \\
\hline Chenopodiaceae & Chenopodium album L. 흰명아주 & 1 \\
\hline Amaranthaceae & Amaranthus patulus Bertol. 가는털비름 & 1 \\
\hline Cruciferae & $\begin{array}{l}\text { Brassica juncea (L.) Czern. 갓, Lepidium apetalum Willd. 다닥냉이, Thlaspi } \\
\text { arvense L. 말냉이 }\end{array}$ & 3 \\
\hline Leguminosae & Trifolium repens L. 토끼풀 & 1 \\
\hline Malvaceae & Abutilon theophrasti Medicus 어저귀 & 1 \\
\hline Violaceae & Viola papilionacea Pursh 종지나물 & 1 \\
\hline Onagraceae & Oenothera biennis L. 달맞이꽃 & 1 \\
\hline Convolvulaceae & $\begin{array}{l}\text { Ipomoea hederacea Jacq. 미국나팔꽃, Ipomoea hederacea var. integriuscula } \\
\text { A.Gray 둥근잎미국나팔꽃 }\end{array}$ & 2 \\
\hline Solanaceae & Datura stramonium var. chalybea Koch. 독말풀 & 1 \\
\hline Scrophulariaceae & Veronica arvensis L. 선개불알풀, Veronica persica Poir. 큰개불알풀 & 2 \\
\hline Compositae & $\begin{array}{l}\text { Ambrosia artemisiifolia L. 돼지풀, Carduus crispus L. 지느러미엉겅퀴, Conyza } \\
\text { canadensis (L.) Cronquist 망초, Conyza sumatrensis E.Walker 큰망초, Coreopsis } \\
\text { lanceolata L. 큰금계국, Cosmos sulphureus Cav. 노랑코스모스, Erigeron } \\
\text { annuus (L.) Pers. 개망초, Senecio vulgaris L. 개쑥갓, Sonchus asper (L.) Hill } \\
\text { 큰방가지똥, Sonchus oleraceus L. 방가지똥, Taraxacum officinale Weber 서양 } \\
\text { 민들레 }\end{array}$ & 11 \\
\hline Gramineae & $\begin{array}{l}\text { Agropyron repens (L.) P.Beauv. 구주개밀, Agropyron repens f. aristatum Holmb. } \\
\text { 까락구주개밀, Bromus rigidus Roth 긴까락빕새귀리, Bromus tectorum L. 털빕 } \\
\text { 새귀리, Cenchrus longispinus (Hack.) Fern. 대청가시풀, Dactylis glomerata L. } \\
\text { 오리새, Festuca arundinacea Schreb. 큰김의털, Festuca myuros L. 들묵새, } \\
\text { Phleum pratense L. 큰조아재비, Poa compressa L. 좀포아풀, Poa pratensis L. } \\
\text { 왕포아풀 }\end{array}$ & 11 \\
\hline
\end{tabular}

40 taxa

편이 모두 억센 가시로 변하여 인간과 동물에게 물리적으 로 영향을 줄 수 있고, 미국에서도 이 Cenchrus속은 가시 가 형성되기 전에는 좋은 목초지만 이후는 문제가 되는 잡 초로 알려져 있다(Hitchcock, 1971). 백사장이나 민가의 농 지와 밭 주변으로 확산될 시 큰 피해를 줄 수 있어 계속적인 모니터링과 제거작업이 이루어져야 할 것으로 생각된다.

\section{적 요}

본 연구는 2008년과 2010년에 걸쳐 3차례 식물상을 조 사하였으며, 전체 조사된 분류군은 90 과 269속 351종 3 아 종 43 변종 6 품종등 403 분류군이 확인되었다. 주요 식물들
로 특산식물이 서울제비꽃 등 2 분류군이 확인되었고, 산림 청지정 희귀 및 멸종위기 식물이 대청부채 $(\mathrm{CR})$ 등 8 분류 군, 환경부 지정 특정식물은 금방망이 $(\mathrm{V})$ 등 35 분류군으 로 나타났다. 또한 식물지리학적 분포특성에 있어 남방계 식물과 북방계식물이 함께 확인되었다. 귀화식물은 대청가 시풀 등 40 분류군이 확인되었으며, 한반도 미기록종인 대 청지치가 새로이 발견되었다.

\section{인용문헌}

Choi, B.H. and J.H. Lee. 2008. The Flora of Coastal Dune Area in Island Dai-chung. 2008 Coastal Dune survey. Ministry 
of Environment and National Institute of Environmental Research. pp. 93-103 (in Korean).

Hitchcock, A.S. 1971. Manual of the Grasses of the United States. Vol. 2. Dover Publication, INC, New York, USA. pp. 730-735.

Korea Meteorological Administration. 2009. Annual Report of Automatic Weather Station Data. Korea Meteorological Administration, Seoul (in Korean).

Korea National Arboretum. 2008. Rare Plants Data Book in Korea. Korea National Arboretum, Pocheon, Korea (in Korean).

Lee, I.K. and H.J. Lee. 1982. Ecological study on the flora of island Dai-chung. The Research Institute of Basic Sciences Kon-Kuk University. J. Basic Sci. 7:31-43 (in Korean).

Lee, T.B. 1980. Illustrated Flora of Korea. Hyangmunsa. Seoul, Korea (in Korean). 2003. Coloured Flora of Korea $(1,2)$. Hyangmunsa. Seoul, Korea. (in Korean).

Lee, W.C. 1996a. Standard Illustrations of Korean plants. Academy Publishing Co. Seoul, Korea (in Korean). 1996b. Lineamenta florae Koreae. Academy Publishing Co. Seoul, Korea (in Korean).
Lee, W.C. and Y.J. Yim. 2002. Plant Geography with Special Reference to Korea. Kangwon National University Press. Chuncheon, Korea (in Korean).

Ministry of Environment. 2006. The 3rd National Natural Environment Research Guidelines. Ministry of Environment and National Institute of Environmental Research, Gwacheon \& Incheon, Korea (in Korean).

Oh, B.U., D.G. Jo, K.S. Kim and C.G. Jang. 2005. Endemic Vascular Plants in the Korean Peninsula. Korea National Arboretum, Pocheon, Korea (in Korean).

Park, S.H. 1995. Colored Illustrations of Naturalized Plants of Korea. Ilchokak, Seoul, Korea (in Korean). . 2001. Colored Illustrations of Naturalized Plants of Korea (appendix). Ilchokak, Seoul, Korea (in Korean). . 2009. New Illustrations and Photographs of

Naturalized Plants of Korea. Ilchokak, Seoul, Korea (in Korean).

Yang, J.C., Y.M. Lee, S.H. Park and S.G. Ha. 2010. The first record of Thyrocarpus glochidiatus (Boraginaceae) in Korea. Korean J. Plant Taxon. 40(1):71-73 (in Korean).

(접수일 2011.1.11; 수정일 2011.6.27; 채택일 2011.11.10) 
Appendix 1. The list of vascular plants in Daecheongdo

Equisetaceae 속새과

Equisetum arvense L. 쇠뜨기 ParkSH80108

Ophioglossaceae 고사리삼과

Sceptridium ternatum (Thunb.) Lyon 고사리삼 ParkSH100033

Osmundaceae 고비과

Osmunda japonica Thunb. 고비 ParkSH80174

Dennstaedtiaceae 잔고사리과

Pteridium aquilinum var. latiusculum (Desv.) Und. ex Heller. 고사리 ParkSH80135

Dryopteridaceae 면마과

Cyrtomium falcatum (L.f.) C.Presl 도깨비쇠고비 Y.81115

Dryopteris bissetiana (Bak.) C.Chr. 산족제비고 사리 ParkSH100068

Dryopteris chinensis (Bak.) Koidz. 가는잎족제비고사리 Y.80629

Dryopteris lacera (Thunb.) Kuntze 비늘고사리 ParkSH80298

Polystichum tripteron (Kunze) C.Presl 십자고사리 ParkSH80190

Thelypteridaceae 처녀고사리과

Thelypteris palustris (Salisb.) Schott 처녀고사리 ParkSH80321

Woodsiaceae 우드풀과

Athyrium niponicum (Mett.) Hance 개고사리 ParkSH80122

Athyrium yokoscense (Franch. \& Sav.) Christ 뱀고사리 ParkSH80180

Deparia japonica (Thunb.) M.Kato 진고사리 ParkSH80295

Onoclea sensibilis var. interrupta Maxim. 야산고비 ParkSH80268

Polypodiaceae 고란초과

Lepisorus ussuriensis (Regel \& Maack) Ching 산일엽초 ParkSH80289

Pinaceae 소나무과

Larix kaempferi (Lamb.) Carriére 일본잎갈나무 ParkSH100049

Pinus densiflora Siebold \& Zucc. 소나무 ParkSH80116

Pinus thunbergii Parl. 곰솔 ParkSH100005

Salicaceae 버드나무과

Salix babylonica L. 수양버들 ParkSH100056

Salix koreensis Andersson 버드나무 ParkSH80087

Betulaceae 자작나무과

Alnus firma Siebold \& Zucc. 사방오리 ParkSH100006

Alnus sibirica Fisch. ex Turcz. 물오리나무 ParkSH80238

Carpinus cordata Blume 까치박달 ParkSH80189

Carpinus turczaninovii Hance 소사나무 ParkSH80125

Corylus heterophylla Fisch. ex Trautv. 개암나무 ParkSH80093

Fagaceae 참나무과

Quercus aliena Blume 갈참나무 ParkSH80141

Quercus dentata Thunb. 떡갈나무 ParkSH80178

Quercus mongolica Fisch. ex Ledeb. 신갈나무 Y.80640

Quercus serrata Thunb. 졸참나무 ParkSH80111

Quercus variabilis Blume 굴참나무 ParkSH80203

Ulmaceae 느릅나무과

Celtis sinensis Pers. 팽나무 ParkSH80208

Ulmus davidiana var. japonica (Rehder) Nakai 느릅나무 ParkSH100023 
Appendix 1. Continued

Moraceae 뽕나무과

Morus alba L. 뽕나무 ParkSH80095

Morus bombycis Koidz. 산뽕나무 ParkSH80036

Cannabaceae 삼과

Humulus japonicus Siebold \& Zucc. 환삼덩굴 Y.81162

Urticaceae 쐐기풀과

Boehmeria longispica Steud. 왜모시풀 Y.81090

Boehmeria sieboldiana Blume 긴잎모시풀 Y.81065

Santalaceae 단향과

Thesium chinense Turcz. 제비꿀 ParkSH80061

Polygonaceae 마디풀과

Fallopia convolvulus (L.) A.Löve 나도닭의덩굴 Y.81089

Persicaria filiformis (Thunb.) Nakai ex Mori 이삭여뀌 Y.81097

Persicaria japonica (Meisn.) H.Gross ex Nakai 흰꽃여뀌 Y.80673

Persicaria lapathifolia var. salicifolia Miyabe 솜흰여뀌 Y.81116

Persicaria longiseta (Bruijn) Kitag. 개여뀌 Y.81164

Persicaria nodosa (Pers.) Opiz 큰개여뀌 Y.81155

Persicaria perfoliata (L.) H.Gross 며느리배꼽 Y.81169

Persicaria senticosa (Meisn.) H.Gross ex Nakai 며느리밑씻개 Y.81110

Rumex acetosa L. 수영 ParkSH80306

Rumex crispus L. 소리쟁이 ParkSH80158

Rumex japonicus Houtt. 참소리쟁이 ParkSH80258

Portulacaceae 쇠비름과

Portulaca oleracea L. 쇠비름 Y.81136

\section{Caryophyllaceae 석죽과}

Arenaria serpyllifolia L. 벼룩이자리 ParkSH80079

Cerastium glomeratum Thuill. 유럽점나도나물 ParkSH80082

Cerastium holosteoides var. hallaisanense (Nakai) Mizush. 점나도나물 ParkSH80274

Cucubalus baccifer var. japonicus Miq. 덩굴별꽃 Y.81070

Gypsophila oldhamiana Miq. 대나물 ParkSH80062

Pseudostellaria heterophylla (Miq.) Pax ex Pax \& Hoffm. 개별꽃 ParkSH100044

Sagina maxima A.Gray 큰개미자리 ParkSH80264

Silene aprica var. oldhamiana (Miq.) C.Y.Wu 갯장구채 ParkSH80150

Silene armeria L. 끈끈이대나물 Y.81154

Silene firma Siebold \& Zucc. 장구채 Y.81118

Stellaria alsine var. undulata (Thunb.) Ohwi 벼룩나물 ParkSH80256

Stellaria aquatica (L.) Scop. 쇠별꽃 ParkSH80285

Stellaria media (L.) Vill. 별꽃 ParkSH100008

Stellaria neglecta Weihe ex Bluff \& Fingerh. 초록별꽃 ParkSH100028

Chenopodiaceae 명아주과

Atriplex gmelinii C.A.Mey. 가는갯는쟁이 Y.81134

Chenopodium album L. 흰명아주 Y.81165

Kochia scoparia (L.) Schrad. 댑싸리 Y.81184

Suaeda glauca (Bunge) Bunge 나문재 Y.81180

Amaranthaceae 비름과

Achyranthes fauriei H.Lév. \& Vaniot 털쇠무릎 Y.81063

Amaranthus patulus Bertol. 가는털비름 Y.81185 
Appendix 1. Continued

Lauraceae 녹나무과

Lindera erythrocarpa Makino 비목나무 ParkSH80254

Lindera glauca (Siebold \& Zucc.) Blume 감태나무 Y.81109

Lindera obtusiloba Blume 생강나무 ParkSH100021

Machilus thunbergii Siebold \& Zucc. 후박나무 Y.81047

Ranunculaceae 미나리아재비과

Clematis heracleifolia DC. 병조희풀 Y.80660

Clematis terniflora var. mandshurica (Rupr.) Ohwi 으아리 Y.81027

Ranunculus chinensis Bunge 젓가락나물 ParkSH80230

Thalictrum kemense var. hypoleucum (Siebold \& Zucc.) Kitag. 좀뀡의다리 Y.80705

Lardizabalaceae 으름덩굴과

Akebia quinata (Thunb.) Decne. 으름덩굴 ParkSH80065

Menispermaceae 새모래덩굴과

Cocculus trilobus (Thunb.) DC. 댕댕이덩굴 Y.81012

Actinidiaceae 다래나무과

Actinidia arguta (Siebold \& Zucc.) Planch. ex Miq. 다래 Y.80620

Theaceae 차나무과

Camellia japonica L. 동백나무 ParkSH80212

Fumariaceae 현호색과

Corydalis turtschaninovii Besser 조선현호색 ParkSH100022

Cruciferae 십자화과

Arabis glabra Bernh. 장대나물 ParkSH80083

Barbarea orthoceras Ledeb. 나도냉이 ParkSH80284

Brassica juncea (L.) Czern. 갓 ParkSH80059

Capsella bursa-pastoris (L.) L.W.Medicus 냉이 ParkSH80034

Cardamine fallax L. 좁쌀냉이 ParkSH100012

Cardamine flexuosa With. 황새냉이 ParkSH80214

Cardamine impatiens L. 싸리냉이 ParkSH80252

Draba nemorosa L. 꽃다지 ParkSH100053

Lepidium apetalum Willd. 다닥냉이 Y.81059

Rorippa palustris (Leyss.) Besser 속속이풀 ParkSH80221

Thlaspi arvense L. 말냉이 ParkSH80217

Platanaceae 버즘나무과

Platanus occidentalis L. 양버즘나무 ParkSH80299

Crassulaceae 돌나무과

Hylotelephium erythrostictum (Miq.) H.Ohba 뀡의비름 ParkSH80195

Orostachys japonicus (Maxim.) A.Berger 바위솔 Y.80703

Penthorum chinense Pursh 낙지다리 ParkSH80328

Sedum kamtschaticum Fisch. \& Mey. 기린초 Y.81032

Saxifragaceae 범의귀과

Ribes fasciculatum var. chinense Maxim. 까마귀밥나무 ParkSH100034

Rosaceae 장미과

Agrimonia pilosa Ledeb. 짚신나물 Y.81043

Duchesnea indica (Andr.) Focke 뱀딸기 ParkSH80033

Malus baccata Borkh. 야광나무 ParkSH80266

Potentilla chinensis Ser. 딱지꽃 Y.81098

Potentilla freyniana Bornm. 세잎양지꽃 ParkSH100035 
Appendix 1. Continued

Prunus persica (L.) Batsch 복사나무 ParkSH80160

Prunus serrulata var. spontanea (Maxim.) E.H.Wilson 벚나무 ParkSH80104

Rhodotypos scandens (Thunb.) Makino 병아리꽃나무 ParkSH80071

Rosa multiflora Thunb. 찔레나무 ParkSH80279

Rosa rugosa Thunb. 해당화 ParkSH80054

Rubus coreanus Miq. 복분자딸기 ParkSH80243

Rubus crataegifolius Bunge 산딸기 ParkSH80294

Rubus hirsutus Thunb. 장딸기 ParkSH80037

Rubus parvifolius L. 멍석딸기 ParkSH80275

Rubus phoenicolasius Maxim. 곰딸기 Y.81073

Sanguisorba officinalis L. 오이풀 Y.80694

Sorbus alnifolia (Siebold \& Zucc.) K.Koch 팥배나무 ParkSH80070

Stephanandra incisa (Thunb.) Zabel 국수나무 ParkSH80163

\section{Leguminosae 콩과}

Aeschynomene indica L. 자귀풀 Y.80675

Albizia julibrissin Durazz. 자귀나무 Y.81091

Caesalpinia decapetala (Roth) Alston 실거리나무 ParkSH80153

Chamaecrista nomame (Siebold) H.Ohashi 차풀 Y.81075

Glycine $\max$ (L.) Merr. 콩 Y.80668

Glycine soja Siebold \& Zucc. 돌콩 Y.81076

Indigofera kirilowii Maxim. ex Palib. 땅비싸리 Y.80708

Kummerowia stipulacea (Maxim.) Makino 둥근매듭풀 Y.80654

Lathyrus japonicus Willd. 갯완두 ParkSH80048

Lespedeza bicolor Turcz. 싸리 Y.81121

Lespedeza cuneata G.Don 비수리 Y.81093

Pueraria lobata (Willd.) Ohwi 칡 Y.81025

Trifolium repens L. 토끼풀 ParkSH80227

Vicia angustifolia var. segetilis (Thuill) K.Koch. 살갈퀴 ParkSH80039

Vigna angularis var. nipponensis (Ohwi) Ohwi \& H.Ohashi 새팥 Y.81068

Oxalidaceae 괭이밥과

Oxalis corniculata L. 괭이밥 ParkSH80027

Oxalis stricta L. 선괭이밥 ParkSH80337

Geraniaceae 쥐손이풀과

Geranium sibiricum L. 쥐손이풀 Y.80635

Geranium thunbergii Siebold \& Zucc. 이질풀 Y.81078

Geranium wilfordii Maxim. 세잎쥐손이 Y.81046

Euphorbiaceae 대극과

Acalypha australis L. 깨풀 Y.80646

Euphorbia ebracteolata Hayata 붉은대극 ParkSH100055

Euphorbia sieboldiana Morren \& Decne. 개감수 ParkSH80196

Sapium japonicum (Siebold \& Zucc.) Pax \& Hoffm. 사람주나무 ParkSH80288

Securinega suffruticosa (Pall.) Rehder 광대싸리 Y.81042

Rutaceae 운향과

Zanthoxylum piperitum (L.) DC. 초피나무 ParkSH80024

Zanthoxylum schinifolium Siebold \& Zucc. 산초나무 Y.81072

Simaroubaceae 소태나무과

Picrasma quassioides (D.Don) Bennett 소태나무 ParkSH80319 
Appendix 1. Continued

Anacardiaceae 옻나무과

Rhus javanica L. 붉나무 ParkSH80222

Aceraceae 단풍나무과

Acer pictum subsp. mono (Maxim.) Ohashi 고로쇠나무 ParkSH80073

Acer tataricum subsp. ginnala (Maxim.) Wesm. 신나무 ParkSH80261

Sabiaceae 나도밤나무과

Meliosma myriantha Siebold \& Zucc. 나도밤나무 ParkSH80239

Balsaminaceae 봉선화과

Impatiens balsamina L. 봉선화 Y.81156

Celastraceae 노박덩굴과

Celastrus orbiculatus Thunb. 노박덩굴 ParkSH80209

Euonymus alatus (Thunb.) Siebold 화살나무 ParkSH80237

Euonymus hamiltonianus Wall. 참빗살나무 Y.81114

Euonymus sachalinensis (F.Schmidt) Maxim. 회나무 ParkSH80156

Staphyleaceae 고추나무과

Euscaphis japonica (Thunb.) Kanitz 말오줌때 Y.81086

Staphylea bumalda DC. 고추나무 ParkSH80155

Vitaceae 포도과

Ampelopsis brevipedunculata (Maxim.) Trautv. 개머루 Y.80657

Cayratia japonica (Thunb.) Gagnep. 거지덩굴 ParkSH80326

Parthenocissus tricuspidata (Siebold \& Zucc.) Planch. 담쟁이덩굴 ParkSH80063

Vitis ficifolia f. glabrata (Nakai) W.T.Lee 청까마귀머루 Y.81005

Vitis flexuosa Thunb. 새머루 Y.80625

Tiliaceae 피나무과

Grewia parviflora Bunge 장구밤나무 Y.81037

Tilia amurensis Rupr. 피나무 ParkSH80157

Malvaceae 아욱과

Abutilon theophrasti Medicus 어저귀 Y.80645

Elaeagnaceae 보리수나무과

Elaeagnus macrophylla Thunb. 보리밥나무 ParkSH100059

Elaeagnus umbellata Thunb. 보리수나무 ParkSH80044

Violaceae 제비꽃과

Viola acuminata Ledeb. 졸방제비꽃 ParkSH80184

Viola albida var. chaerophylloides (Regel) F.Maek. 남산제비꽃 ParkSH100045

Viola collina Besser 둥근털제비꽃 ParkSH100017

Viola keiskei Miq. 잔털제비꽃 Y.81007

Viola lactiflora Nakai 흰젖제비꽃 ParkSH100047

Viola mandshurica W.Becker 제비꽃 ParkSH100018

Viola papilionacea Pursh 종지나물 ParkSH100043

Viola rossii Hemsl. 고깔제비꽃 ParkSH100048

Viola selkirkii Pursh ex Goldie 뫼제비꽃 ParkSH100052

Viola seoulensis Nakai 서울제비꽃 ParkSH100030

Viola yedoensis Makino 호제비꽃 ParkSH100003

Cucurbitaceae 박과

Trichosanthes kirilowii Maxim. 하늘타리 Y.81111

Lythraceae 부처꽃과

Lythrum anceps (Koehne) Makino 부처꽃 Y.81141 
Appendix 1. Continued

Trapaceae 마름과

Trapa japonica Flerow 마름 Y.80681

Onagraceae 바늘꽃과

Circaea cordata Royle 쇠털이슬 Y.81069

Oenothera biennis L. 달맞이꽃 Y.81045

Araliaceae 두릅나무과

Aralia elata (Miq.) Seem. 두릅나무 Y.81003

Eleutherococcus sessiliflorus (Rupr. \& Maxim.) S.Y.Hu 오갈피나무 ParkSH80311

Kalopanax septemlobus (Thunb.) Koidz. 음나무 Y.80664

Umbelliferae 산형과

Angelica cartilaginomarginata (Makino) Nakai 처녀바디 Y.80697

Cnidium japonicum Miq. 갯사상자 Y.81108

Oenanthe javanica (Blume) DC. 미나리 Y.81101

Ostericum grosseserratum (Maxim.) Kitag. 신감채 Y.80618

Peucedanum terebinthaceum (Fisch.) Fisch. ex DC. 기름나물 Y.81030

Torilis japonica (Houtt.) DC. 사상자 Y.81062

Ericaceae 진달래과

Rhododendron mucronulatum Turcz. 진달래 ParkSH100019

Primulaceae 앵초과

Androsace umbellata (Lour.) Merr. 봄맞이 ParkSH80255

Lysimachia clethroides Duby 큰까치수염 Y.81083

Styracaceae 때죽나무과

Styrax japonicus Siebold \& Zucc. 때죽나무 ParkSH80185

Symplocaceae 노린재나무과

Symplocos chinensis f. pilosa (Nakai) Ohwi 노린재나무 ParkSH80102

Oleaceae 물푸레나무과

Forsythia koreana (Rehder) Nakai 개나리 ParkSH80072 (식재)

Ligustrum obtusifolium Siebold \& Zucc. 쥐똥나무 ParkSH80099

Gentianaceae 용담과

Gentiana zollingeri Faw. 큰구슬붕이 ParkSH100037

Apocynaceae 협죽도과

Amsonia elliptica (Thunb.) Roem. \& Schult. 정향풀 ParkSH80210

Asclepiadaceae 박주가리과

Metaplexis japonica (Thunb.) Makino 박주가리 Y.81092

Rubiaceae 꼭두서니과

Galium pogonanthum Franch. \& Sav. 산갈퀴 ParkSH80186

Galium spurium var. echinospermum (Wallr.) Hayek 갈퀴덩굴 ParkSH80043

Rubia cordifolia var. pratensis Maxim. 갈퀴꼭두서니 ParkSH80031

Convolvulaceae 메꽃과

Calystegia hederacea Wall. 애기메꽃 Y.81036

Ipomoea hederacea Jacq. 미국나팔꽃 Y.80636

Ipomoea hederacea var. integriuscula A.Gray 둥근잎미국나팔꽃 Y.80684

Boraginaceae 지치과

Bothriospermum tenellum (Hornem.) Fisch. \& C.A.Mey. 꽃받이 ParkSH80272

Lithospermum arvense L. 개지치 ParkSH100058

Lithospermum zollingeri A.DC. 반디지치 ParkSH80115

Thyrocarpus glochidiatus Maxim. 대청지치 ParkSH100054 
Appendix 1. Continued

Trigonotis peduncularis (Trevir.) Benth. ex Hemsl. 꽃마리 ParkSH100026

Verbenaceae 마편초과

Callicarpa japonica Thunb. 작살나무 Y.81132

Clerodendrum trichotomum Thunb. 누리장나무 Y.81144

Vitex rotundifolia L.f. 순비기나무 Y.81189

Labiatae 꿀풀과

Agastache rugosa (Fisch. \& Mey.) Kuntze 배초향 Y.81016

Clinopodium chinense var. parviflorum (Kudô) Hara 층층이꽃 Y.81040

Clinopodium gracile var. multicaule (Maxim.) Ohwi 탑꽃 Y.81048

Isodon inflexus (Thunb.) Kudô 산박하 Y.80711

Lamium amplexicaule L. 광대나물 ParkSH100009

Leonurus japonicus Houtt. 익모초 Y.81014

Lycopus lucidus Turcz. 쉽싸리 Y.81142

Prunella vulgaris var. lilacina Nakai 꿀풀 Y.80655

Scutellaria pekinensis var. transitra (Makino) Hara 산골무꽃 Y.80656

Scutellaria strigillosa Hemsl. 참골무꽃 Y.81188

Teucrium viscidum var. miquelianum (Maxim.) Hara 덩굴곽향 Y.80666

Solanaceae 가지과

Datura stramonium var. chalybea Koch. 독말풀 Y.80719

Solanum nigrum L. 까마중 Y.81168

Scrophulariaceae 현삼과

Mazus pumilus (Burm.f.) Steenis 주름잎 ParkSH100016

Melampyrum roseum Maxim. 꽃며느리밥풀 Y.81002

Siphonostegia chinensis Benth. 절국대 Y.81022

Veronica arvensis L. 선개불알풀 ParkSH80226

Veronica didyma var. lilacina (H.Hara) T.Yamaz. 개불알풀 ParkSH100027

Veronica linariifolia Pall. ex Link 꼬리풀 Y.80695

Veronica persica Poir. 큰개불알풀 ParkSH80042

Phrymaceae 파리풀과

Phryma leptostachya var. asiatica H.Hara 파리풀 Y.81079

Plantaginaceae 질경이과

Plantago asiatica L. 질경이 ParkSH80281

Plantago camtschatica Cham. ex Link 개질경이 ParkSH80134

\section{Caprifoliaceae 인동과}

Lonicera japonica Thunb. 인동 Y.80692

Lonicera japonica var. repens (Siebold) Rehder 털인동 Y.81006

Viburnum carlesii Hemsl. 분꽃나무 ParkSH100067

Viburnum erosum Thunb. 덜뀡나무 ParkSH80089

Weigela praecox (Lemoine) L.H.Bailey 소영도리나무 ParkSH80152

Weigela subsessilis (Nakai) L.H.Bailey 병꽃나무 Y.80713

Valerianaceae 마타리과

Patrinia scabiosaefolia Fisch. ex Trevir. 마타리 Y.81080

Patrinia villosa (Thunb.) Juss. 뚝갈 Y.81020

Campanulaceae 초롱꽃과

Codonopsis lanceolata (Siebold \& Zucc.) Trautv. 더덕 ParkSH80161 
Appendix 1. Continued

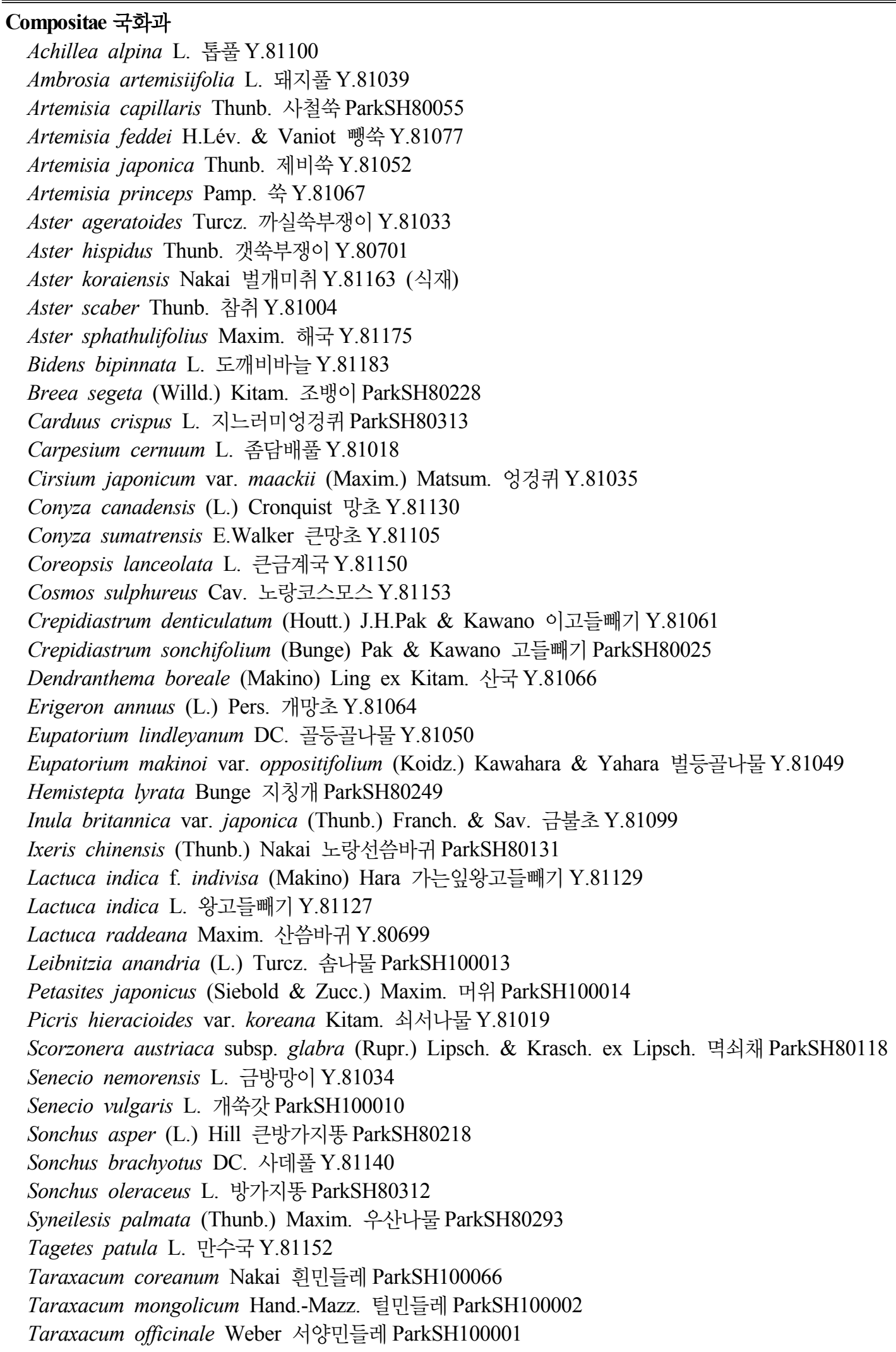


Appendix 1. Continued

Taraxacum platycarpum Dahlst. 민들레 ParkSH80103

Tephroseris kirilowii (Turcz. ex DC.) Holub 솜방망이 ParkSH100046

Youngia japonica (L.) DC. 뽀리뱅이 ParkSH80213

Potamogetonaceae 가래과

Potamogeton distinctus A.Benn. 가래 Y.80672

Liliaceae 백합과

Allium anisopodium Ledeb. 실부추 ParkSH80113

Allium tenuissimum L. 애기실부추 Y.81172

Allium macrostemon Bunge 산달래 ParkSH80074

Asparagus oligoclonos Maxim. 방울비짜루 ParkSH80168

Asparagus schoberioides Kunth 비짜루 ParkSH80075

Disporum smilacinum A.Gray 애기나리 ParkSH80091

Lilium lancifolium Thunb. 참나리 Y.81106

Liriope spicata (Thunb.) Lour. 개맥문동 Y.81038

Polygonatum humile Fisher. ex Maxim. 각시둥굴레 ParkSH80296

Polygonatum odoratum var. pluriflorum (Miq.) Ohwi 둥굴레 ParkSH80278

Scilla scilloides (Lindl.) Druce 무릇 Y.81124

Smilacina japonica A.Gray 풀솜대 ParkSH80198

Smilax china L. 청미래덩굴 ParkSH80094

Smilax nipponica Miq. 선밀나물 ParkSH80069

Smilax sieboldii Miq. 청가시덩굴 ParkSH80151

Tulipa edulis (Miq.) Baker 산자고 ParkSH100063

Dioscoreaceae 마과

Dioscorea batatas Decne. 마 Y.81055

Pontederiaceae 물옥잠과

Monochoria vaginalis var. plantaginea (Roxb.) Solms 물달개비 Y.80674

Iridaceae 붓꽃과

Belamcanda chinensis (L.) DC. 범부채 Y.81024

Iris dichotoma Pall. 대청부채 Y.80714

Juncaceae 골풀과

Juncus diastrophanthus Buchenau 별날개골풀 Y.81145

Juncus setchuensis var. effusoides Buchenau 푸른갯골풀 Y.81051

Luzula capitata (Miq.) Miq. 뀡의밥 ParkSH100004

Commelinaceae 닭의장풀과

Commelina communis L. 닭의장풀 Y.81159

Gramineae 벼과

Agropyron repens (L.) P.Beauv. 구주개밀 ParkSH80332

Agropyron repens f. aristatum Holmb. 까락구주개밀 ParkSH80331

Alopecurus aequalis var. amurensis (Kom.) Ohwi 뚝새풀 ParkSH80026

Arundinella hirta var. ciliata Koidz. 털새 Y.81071

Bromus japonicus Thunb. 참새귀리 ParkSH80028

Bromus pauciflorus (Thunb.) Hack. 꼬리새 Y.81017

Bromus rigidus Roth 긴까락빕새귀리 ParkSH80030

Bromus tectorum L. 털빕새귀리 ParkSH80040

Calamagrostis epigeios (L.) Roth 산조풀 ParkSH80329

Cenchrus longispinus (Hack.) Fern. 대청가시풀 Y.80718 
Appendix 1. Continued

Cynodon dactylon (L.) Pers. 우산잔디 Y.80690

Dactylis glomerata L. 오리새 ParkSH80038

Digitaria ciliaris (Retz.) Koel. 바랭이 Y.80679

Echinochloa crusgalli (L.) P.Beauv. 돌피 Y.81161

Eleusine indica (L.) Gaertn. 왕바랭이 Y.81186

Elymus dahuricus Turcz. ex Griseb. 갯보리 Y.81095

Elymus mollis Trin. 갯그령 ParkSH80050

Eragrostis ferruginea (Thunb.) P.Beauv. 그령 Y.81053

Eriochloa villosa (Thunb.) Kunth 나도개피 Y.81081

Festuca arundinacea Schreb. 큰김의털 ParkSH80078

Festuca myuros L. 들묵새 Y.80643

Festuca parvigluma Steud. 김의털아재비 ParkSH80205

Hierochloe odorata (L.) P.Beauv. 향모 ParkSH100041

Imperata cylindrica var. koenigii (Retz.) Pilg. 띠 ParkSH80123

Ischaemum anthephoroides (Steud.) Miq. 갯쇠보리 Y.80687

Ischaemum crassipes (Steud.) Thell. 쇠보리 Y.81178

Melica onoei Franch. \& Sav. 쌀새 Y.81060

Oplismenus undulatifolius var. japonicus (Steud.) Koidz. 민주름조개풀 Y.80665

Paspalum thunbergii Kunth ex Steud. 참새피 Y.81094

Pennisetum alopecuroides (L.) Spreng. 수크령 Y.81074

Phleum pratense L. 큰조아재비 Y.81122

Phragmites communis Trin. 갈대 ParkSH80107

Poa acroleuca Steud. 실포아풀 ParkSH80335

Poa annua L. 새포아풀 ParkSH100011

Poa compressa L. 좀포아풀 Y.80670

Poa pratensis L. 왕포아풀 ParkSH80077

Poa sphondylodes Trin. 포아풀 ParkSH80051

Pseudosasa japonica (Siebold \& Zucc. ex Steud.) Makino 이대 ParkSH100007

Sasa borealis (Hack.) Makino 조릿대 ParkSH80232

Setaria faberii Herrm. 가을강아지풀 Y.81158

Setaria glauca (L.) P.Beauv. 금강아지풀 Y.81138

Setaria pallidefusca (Schumach.) Stapf \& C.E.Hubb. 가는금강아지풀 Y.81044

Setaria viridis (L.) P.Beauv. 강아지풀 Y.81151

Spodiopogon sibiricus Trin. 큰기름새 Y.81028

Sporobolus japonicus (Steud.) Maxim. ex Rendle 나도잔디 ParkSH80112

Stipa pekinensis Hance 나래새 Y.81088

Themeda triandra var. japonica (Willd.) Makino 솔새 ParkSH80127

Zizania latifolia (Griseb.) Turcz. ex Stapf 줄 Y.80680

Zoysia japonica Steud. 잔디 ParkSH80132

Araceae 천남성과

Arisaema amurense Maxim. 둥근잎천남성 ParkSH100029

Arisaema amurense f. serratum (Nakai) Kitagausa 천남성 ParkSH80045

Arisaema heterophyllum Blume 두루미천남성 ParkSH80149

Arisaema ringens (Thunb.) Schott 큰천남성 ParkSH80120

Pinellia ternata (Thunb.) Breitenb. 반하 ParkSH80121 
Appendix 1. Continued

\section{Cyperaceae 사초과}

Bulbostylis densa (Wall.) Hand.-Mazz. 꽃하늘지기 Y.80669

Carex bostrychostigma Maxim. 길뚝사초 ParkSH80179

Carex breviculmis R.Br. 청사초 ParkSH80080

Carex breviculmis var. fibrillosa Kük. 갯청사초 ParkSH80053

Carex forficula Franch. \& Sav. 산뚝사초 ParkSH100036

Carex humilis var. nana (H.Lév. \& Vaniot) Ohwi 가는잎그늘사초 ParkSH80140

Carex japonica Thunb. 개찌버리사초 ParkSH80250

Carex kobomugi Ohwi 통보리사초 ParkSH80052

Carex laevissima Nakai 애괭이사초 ParkSH80128

Carex lanceolata Boott 그늘사초 ParkSH100025

Carex neurocarpa Maxim. 괭이사초 Y.81107

Carex polyschoena H.Lév. \& Vaniot 가지청사초 ParkSH80064

Carex pumila Thunb. 좀보리사초 ParkSH80047

Carex sabynensis Less. ex Kunth 실청사초 ParkSH80114

Carex siderosticta Hance 대사초 ParkSH100032

Cyperus difformis L. 알방동사니 Y.81160

Cyperus microiria Steud. 금방동사니 Y.81157

Fimbristylis complanata f. exalata T.Koyama 들하늘지기 Y.80717

Fimbristylis dichotoma (L.) Vahl 하늘지기 Y.81056

Fimbristylis tristachya var. subbispicata (Nees \& Meyen) T.Koyama 꼴하늘지기 Y.81058

Kyllinga brevifolia Rottb. 파대가리 Y.81054

Orchidaceae 난과

Cymbidium goeringii (Rchb.f.) Rchb.f. 보춘화 ParkSH100024

Epipactis thunbergii A.Gray 닭의난초 Y.80706

Platanthera freynii Kraenzl. 제비난초 ParkSH80096

Platanthera mandarinorum var. brachycentron (Franch. \& Sav.) Koidz. ex Ohwi 산제비란 Y.80671

Spiranthes sinensis (Pers.) Ames 타래난초 Y.80616 